\title{
The Efficacy And Safety of Anlotinib Combined With Platinum-Etoposide Chemotherapy As First-Line Treatment For Extensive-Stage Small Cell Lung Cancer: A Chinese Multicenter Real-World Study
}

\section{Hao-Ran Zheng}

The First Affiliated Hospital of Xi'an Jiaotong University https://orcid.org/0000-0002-4221-3085

Ai-Min Jiang

The First Affiliated Hospital of Xi'an Jiaotong University

\section{Huan Gao}

The First Affiliated Hospital of Xi'an Jiaotong University

$\mathrm{Na}$ Liu

The First Affiliated Hospital of Xi'an Jiaotong University

Xiao-Qiang Zheng

The First Affiliated Hospital of Xi'an Jiaotong University

\section{Xiao Fu}

The First Affiliated Hospital of Xi'an Jiaotong University

\section{Zhi-Ping Ruan}

The First Affiliated Hospital of Xi'an Jiaotong University

\section{Tao Tian}

The First Affiliated Hospital of Xi'an Jiaotong University

\section{Xuan Liang}

The First Affiliated Hospital of Xi'an Jiaotong University

Yu Yao ( 13572101611@163.com )

The First Affiliated Hospital of Xi'an Jiaotong University https://orcid.org/0000-0003-3618-2363

\section{Research Article}

Keywords: Small cell lung cancer, Anlotinib, Chemotherapy, Real-world data

Posted Date: January 21st, 2022

DOI: https://doi.org/10.21203/rs.3.rs-1271045/v1

License: (9) This work is licensed under a Creative Commons Attribution 4.0 International License. 
Page $2 / 22$ 


\section{Abstract}

Background: Anlotinib, an antiangiogenic multi-target tyrosine kinase inhibitor (TKI), was approved as the third-line and above treatment for small cell lung cancer (SCLC) in China. Some small sample size clinical trials have shown the favorable efficacy of anlotinib combined with platinum-etoposide chemotherapy as the first-line treatment for extensive-stage SCLC (ES-SCLC). This research aimed to explore the real-world efficacy and safety of anlotinib combined with platinum-etoposide chemotherapy.

Methods: Pathologically confirmed ES-SCLC patients receiving anlotinib plus platinum-etoposide chemotherapy as the first-line treatment were enrolled in this retrospective study. The primary endpoint of this study was progression-free survival (PFS), and secondary endpoints included overall survival (OS), objective response rate (ORR), disease control rate (DCR), and adverse reactions.

Results: In total, 58 patients were included in this study. The median PFS was 6.0 months [95\% confidence interval (CI): 3.5-8.5], and the median OS was 10.5 months ( $95 \% \mathrm{Cl} 8.7-12.3)$. Thirty-four patients achieved partial response (PR), 18 patients achieved stable disease (SD), and 6 patients achieved progressive disease (PD). Respectively, the ORR and DCR were $58.6 \%$ and $89.6 \%$. The main treatment-related adverse reactions were generally tolerated. The most common adverse reaction was myelosuppression (44.8\%), followed by hypertension (41.4\%), fatigue (34.5\%), gastrointestinal reaction (32.7\%), hand-foot syndrome (24.1\%), and so on. Multivariate analysis showed that post-medication hand-foot syndrome [PFS 8.5 vs. 5.5 months, Hazards Ratio $(\mathrm{HR})=0.23,95 \% \mathrm{Cl} 0.07-0.72, P=0.012$ ] was the independent predictor of PFS, and hypertension (OS 15.9 vs. 8.3 months, $\mathrm{HR}=0.18,95 \% \mathrm{Cl} 0.05-0.58, P$ $=0.005$ ) was the independent predictor of OS.

Conclusion: Anlotinib combined with platinum-etoposide chemotherapy as the first-line treatment for ESSCLC appears to be effective and well-tolerated in the real-world. Patients with post-medication hypertension and hand-foot syndrome may confer superior prognosis. Further investigations are needed by prospective studies with larger sample size.

\section{Introduction}

Lung cancer is the most frequent cause of tumor death worldwide. Small cell lung cancer (SCLC) is a highly aggressive and deadly malignant tumor, accounting for approximately $10-15 \%$ of all lung cancers $^{[1]}$. SCLC comprises an estimated 250,000 new cases and at least 200,000 deaths worldwide each year ${ }^{[2]}$. Approximately $70 \%$ of the patients are diagnosed with extensive-stage SCLC (ES-SCLC) with poor overall survival (OS) ${ }^{[3]}$. It has been reported that the median OS for ES-SCLC patients without systemic therapy is 2 to 4 months ${ }^{[4,5]}$.

As the gold standard for SCLC therapy, platinum-etoposide chemotherapy has been widely used in the past 40 years. The median progression-free survival (PFS) of platinum-etoposide chemotherapy as the first-line treatment is about 5 months, and the median OS is about 10 months ${ }^{[6]}$. In recent years, the rapid 
rise of immunotherapy has broken the unshakable position of platinum-etoposide chemotherapy. Atezolizumab, a programmed death-ligand 1 (PD-L1) inhibitor, was studied in IMpower133 clinical trial in combination with platinum-etoposide chemotherapy as the first-line treatment for ES-SCLC. The combined regimen brought survival benefits: the median OS was prolonged for 2 months (12.3 vs. 10.3 months), and the 1-year OS rate was increased by $13.5 \%$ (51.7\% vs. $38.2 \%)$ compared with platinumetoposide chemotherapy ${ }^{[7]}$. Durvalumab, another PD-L1 inhibitor, was also found to have a similar OS benefit (13.0 vs. 10.3 months) in CASPIAN clinical trial ${ }^{[8]}$. PD-L1 plus platinum-etoposide chemotherapy has become the new first-line therapy for ES-SCLC.

Angiogenesis is a complex process that plays an essential role in tumor growth, invasion and metastasis. Vascular endothelial growth factor (VEGF) is the most critical proangiogenic protein ${ }^{[9]}$. Previous studies found that about $80 \%$ of SCLC tissues were positive for VEGF expression, and the VEGF level was an independent prognostic factor in SCLC ${ }^{[10]}$. As an oral antiangiogenic tyrosine kinase inhibitor (TKI), anlotinib targets vascular endothelial growth factor receptor (VEGFR), platelet-derived growth factor receptors (PDGFR), fibroblast growth factor receptor (FGFR), and c-kit ${ }^{[11]}$. Based on ALTER 1202 study, anlotinib was approved by the China Food and Drug Administration (CFDA) in 2019 as the third-line and above treatment for SCLC ${ }^{[12]}$. Additionally, some small sample size clinical trials in China have shown the favorable efficacy of anlotinib combined with platinum-etoposide chemotherapy ${ }^{[13-15]}$. The 2021 American society of clinical oncology (ASCO) meeting announced the preliminary result of a phase II clinical study on the efficacy and safety of anlotinib combined with platinum-etoposide chemotherapy in the first-line treatment of ES-SCLC. Twenty patients could evaluate the efficacy, of which the median PFS was 10.0 months, the median OS was 15.0 months, the objective response rate (ORR) was $90 \%$, and the disease control rate (DCR) was $100 \%{ }^{[13]}$. It was significantly higher than that of traditional chemotherapy.

In clinical trials, patients are strictly screened. Patients with poor conditions, such as the elderly, combined brain metastases, and the Eastern Cooperative Oncology Group Performance Status (ECOG PS) $\geq 2$, are often excluded. Therefore, we conducted this multi-center research to investigate the real-world efficacy and safety of anlotinib combined with platinum-etoposide chemotherapy as the first-line treatment for ES-SCLC.

\section{Methods}

\section{Study design and patients}

This research is a multicenter, non-intervention, retrospective real-world study. ES-SCLC patients receiving anlotinib combined with platinum-etoposide chemotherapy as the first-line treatment in the First Affiliated Hospital of Xi'an Jiaotong University, Xijing Hospital of Air Force Military Medical University, Xianyang Central Hospital, Shaanxi Nuclear Industry 215 Hospital, Hanzhong Central Hospital, and Baoji Traditional Chinese Medicine Hospital were eligible for retrospective analysis between December 1, 2018, and July 31,2021 . These tertiary hospitals are located in Shaanxi, China. The characteristics of patients were 
collected, including age, sex, smoking status, ECOG PS, age-adjusted Charlson comorbidity index (aCCI), TNM stage, number and location of metastases, anlotinib initial dose, imaging and laboratory examination, and adverse reaction.

\section{Inclusion and exclusion criteria}

The inclusion criteria for patients were as follows: (1) age $\geq 18$ years; (2) patients with ES-SCLC diagnosed by pathology have measurable lesions according to the Response Evaluation Criteria in Solid Tumors (RECIST) version 1.1 standard; (3) receiving anlotinib combined with platinum-etoposide chemotherapy as the first-line treatment; (4) ECOG PS $\leq 2$; (5) without surgery. The exclusion criteria for patients were as follows: (1) severe lack of clinical records or loss of follow-up; (2) imaging efficacy evaluation cannot be performed; (3) patients with active bleeding or serious systemic diseases.

\section{Therapeutic methods}

Each patient was treated with 2 to 821 -day cycles of anlotinib $(12 \mathrm{mg} / 10 \mathrm{mg}$, day 1 to 14 of each cycle), etoposide $\left(100 \mathrm{mg} / \mathrm{m}^{2}\right.$ of body surface area, day 1 to 3 of each cycle), and carboplatin (area under the curve of $5 \mathrm{mg} / \mathrm{mL} / \mathrm{min}$, day 1 of each cycle) or cisplatin $\left(25 \mathrm{mg} / \mathrm{m}^{2}\right.$ of body surface area, day 1 to 3 of each cycle), followed by anlotinib maintenance every 3 weeks. The actual dosage was adjusted by qualified physicians according to patients' situation. Treatment was continued until disease progression, death, or unacceptable toxicity.

\section{Efficacy and safety evaluation}

According to the RECIST version 1.1 standard, two qualified physicians independently evaluated the efficacy through computed tomography (CT) or magnetic resonance imaging (MRI). The responses were classified as complete response (CR), partial response (PR), stable disease (SD), or progressive disease (PD). When there was disagreement on the assessment, a third physician was requested to reevaluate. Follow-up data were collected up to October 31, 2021. PFS was defined as the time from the start of treatment until tumor progression or death from any cause before disease progression. OS was defined as the time from the treatment initiation to death. Respectively, ORR or DCR was calculated as the addition of CRs plus PRs or CRs plus PRs plus SDs. The adverse reactions were graded according to the Common Terminology Criteria for Adverse Events (CTCAE) version 4.0. The primary endpoint of this study was PFS, and secondary endpoints included OS, ORR, DCR, and adverse reactions.

\section{Statistical analysis}

Patients' baseline characteristics were summarized as proportions for categorical variables and medians (range) for continuous variables as appropriate. The median PFS, OS, and 95\% confidence interval (Cl) were estimated using the Kaplan-Meier method. Cox proportional hazards regression was used for the univariable and multivariable analyses and to calculate the hazard ratios (HR) with $95 \%$ Cls. All statistical analyses in this study were performed using SPSS version 18.0 for Windows 64.0 and GraphPad Prism version 6.0. A two-tailed $P$-value $<0.05$ was considered statistically different. 


\section{Results}

\section{Baseline clinical characteristics of patients}

In total, 58 patients were included in the present study. The median follow-up duration was 7.9 months. Details of the patients' baseline clinical characteristics were shown in Table 1. The median age of the patients was 59 years (range, 36 to 81 years). A total of 47 patients were male (81.0\%). Former smokers and non smokers were noted in 41 (70.7\%) and 17 (29.3\%) patients. ECOG PS 0-1 were observed in 38 (65.5\%) patients. Forty-three (74.1\%) patients were initially diagnosed in the TNM IV stage. Among them, $24(41.4 \%)$ patients received radiotherapy during the treatment. In addition, patients with post-medication hypertension and hand-foot syndrome were observed in 24 (41.4\%) cases and 14 (24.1\%) cases, respectively. 
Table 1

Baseline clinical characteristics of patients.

\section{Characteristics}

Age (years)

Median (range)

$<65$

$\geq 65$

Sex

Male

Female

Smoking status

Ever

Never

ECOG PS

0-1

2

$\mathrm{aCCl}$

$<8$

$\geq 8$

TNM stage

III

IV

T stage

T1-2

T3-4

$\mathrm{N}$ stage

N0-2

N3

Number of metastatic sites

$<2$
$N(\%)$

$59(36-81)$

41 (70.7)

17 (29.3)

47 (81.0)

$11(19.0)$

$41(70.7)$

17 (29.3)

38 (65.5)

$20(34.5)$

26 (44.8)

$32(55.2)$

15 (25.9)

$43(74.1)$

$23(39.7)$

$35(60.3)$

9 (15.5)

49 (84.5)

$33(56.9)$ 


\begin{tabular}{|c|c|}
\hline Characteristics & $\mathbf{N}(\%)$ \\
\hline$\geq 2$ & $25(43.1)$ \\
\hline \multicolumn{2}{|c|}{ Brain metastases } \\
\hline Yes & $5(8.6)$ \\
\hline No & $53(91.4)$ \\
\hline \multicolumn{2}{|c|}{ Hepatic metastases } \\
\hline Yes & $16(27.6)$ \\
\hline No & $42(72.4)$ \\
\hline \multicolumn{2}{|c|}{ Osseous metastases } \\
\hline Yes & $14(24.1)$ \\
\hline No & $44(75.9)$ \\
\hline \multicolumn{2}{|c|}{ Pleural metastases / pleural effusion } \\
\hline Yes & $20(34.5)$ \\
\hline No & $38(65.5)$ \\
\hline \multicolumn{2}{|c|}{ Lung metastases } \\
\hline Yes & $19(32.8)$ \\
\hline No & $39(67.2)$ \\
\hline \multicolumn{2}{|l|}{ Baseline NSE } \\
\hline$\leq 20 \mathrm{ng} / \mathrm{ml}$ & $19(32.8)$ \\
\hline$>20 \mathrm{ng} / \mathrm{ml}$ & $39(67.2)$ \\
\hline \multicolumn{2}{|c|}{ Anlotinib initial dose } \\
\hline $10 \mathrm{mg}$ & $4(6.9)$ \\
\hline $12 \mathrm{mg}$ & $54(93.1)$ \\
\hline \multicolumn{2}{|c|}{ Plus radiotherapy } \\
\hline Yes & $24(41.4)$ \\
\hline No & $34(58.6)$ \\
\hline \multicolumn{2}{|c|}{ Post-medication hypertension } \\
\hline Yes & $24(41.4)$ \\
\hline No & $34(58.6)$ \\
\hline
\end{tabular}




\section{Characteristics}

Post-medication hand-foot syndrome

Yes

No

\section{$N(\%)$}

$14(24.1)$

$44(75.9)$

Abbreviations: ECOG PS: Eastern Cooperative Oncology Group Performance Status; aCCl: ageadjusted Charlson comorbidity index; NSE: neuron specific enolase.

\section{Clinical efficacy}

The median PFS was 6.0 months $(95 \% \mathrm{Cl} 3.5-8.5)$, and the median OS was 10.5 months $(95 \% \mathrm{Cl} 8.7-12.3)$ (Figure $1 \mathrm{~A}$ and Figure 1B). The 6-month PFS rate was $47.9 \%$, the 6 -month 0 S rate was $72.5 \%$, and the 1year OS rate was $28.9 \%$. Among them, 34 (58.6\%) patients achieved PR, 18 (31.0\%) patients achieved SD, and $6(10.4 \%)$ patients achieved PD. Respectively, the ORR and DCR were $58.6 \%$ and $89.6 \%$. The waterfall plot of tumor best response compared with measurable baseline lesions was shown in Figure 2. A 52year old female patient without metastasis reached the longest PFS of 16.8 months.

Univariate analysis (Table 2) showed that female (9.3 vs. 5.5 months, $P=0.002)$, ECOG PS 0-1 (8.5 vs. 3.1 months, $P<0.001)$, aCCl <8 (8.0 vs. 5.5 months, $P=0.044)$, T1-2 (8.5 vs. 5.4 months, $P=0.007)$, no hepatic metastases ( 8.0 vs. 4.7 months, $P=0.010)$, baseline neuron specific enolase (NSE) $\leq 20 \mathrm{ng} / \mathrm{ml}(8.5 \mathrm{vs} .5 .4$ months, $P=0.006)$, plus radiotherapy ( $8.3 \mathrm{vs} .4 .2$ months, $P=0.002)$, post-medication hypertension ( 8.5 vs. 5.4 months, $P=0.008)$, and post-medication hand-foot syndrome $(8.5 \mathrm{vs} .5 .5$ months, $P=0.040)$ might have longer PFS benefits. Age <65 (15.0 vs. 8.3 months, $P=0.005)$, female (16.8 vs. 9.1 months, $P$ $=0.009$ ), never smoking (16.8 vs. 9.1 months, $P=0.024)$, ECOG PS 0-1 (15.0 vs. 4.0 months, $P<0.001)$, $\mathrm{aCCl}<8$ (15.9 vs. 8.5 months, $P=0.013)$, N0-2 (17.5 vs. 9.2 months, $P=0.043)$, no hepatic metastases (15.0 vs. 5.4 months, $P<0.001$ ), plus radiotherapy (16.8 vs. 7.7 months, $P<0.001)$, and post-medication hypertension (15.9 vs. 8.3 months, $P<0.001)$ might have longer $O S$ benefits. Factors with $P<0.050$ in univariate analyses were included in multivariate Cox regression analysis. Multivariate analysis revealed that sex (male vs. female: $\mathrm{HR}=6.05,95 \% \mathrm{Cl} 1.74-20.98, P=0.005)$, ECOG PS $(2$ vs. $0-1: \mathrm{HR}=8.34,95 \% \mathrm{Cl}$ 2.54-27.39, $P<0.001)$, T stage (T3-4 vs. T1-2: HR=3.82, 95\% Cl 1.59-9.18, $P=0.003)$, and post-medication hand-foot syndrome (yes vs. no: $\mathrm{HR}=0.23,95 \% \mathrm{Cl} 0.07-0.72, P=0.012$ ) were the independent predictors of PFS (Table 3). Age ( $\geq 65$ vs. $<65$ : HR=4.87, 95\%Cl 1.71-13.82, $P=0.003)$, ECOG PS ( 2 vs. $0-1: \mathrm{HR}=11.26$, $95 \% \mathrm{Cl} 2.49-50.84, P=0.002$ ), hepatic metastases (yes vs. no: $\mathrm{HR}=3.83,95 \% \mathrm{Cl} 1.41-10.41, P=0.008$ ), and post-medication hypertension (yes $v s$. no: $\mathrm{HR}=0.18,95 \% \mathrm{Cl} 0.05-0.58, P=0.005$ ) were the independent predictors of OS (Table 4). The Kaplan-Meier curves of PFS and OS in multivariate Cox regression analysis were presented in Figure 3 and Figure 4. 
Table 2

Univariate analysis of factors associated with PFS and OS.

\begin{tabular}{|c|c|c|c|c|c|c|}
\hline Factors & $\begin{array}{l}\text { mPFS } \\
\text { (months) }\end{array}$ & $\begin{array}{l}95 \% \\
\mathrm{Cl}\end{array}$ & $\begin{array}{l}P \\
\text { value }\end{array}$ & $\begin{array}{l}\text { mOS } \\
\text { (months) }\end{array}$ & $95 \% \mathrm{Cl}$ & $\begin{array}{l}P \\
\text { value }\end{array}$ \\
\hline Age (years) & & & 0.262 & & & 0.005 \\
\hline$<65$ & 6.0 & $\begin{array}{l}3.3- \\
8.7\end{array}$ & & 15.0 & $\begin{array}{l}9.8^{-} \\
20.2\end{array}$ & \\
\hline$\geq 65$ & 5.9 & $\begin{array}{l}2.7- \\
9.1\end{array}$ & & 8.3 & 7.6-9.0 & \\
\hline Sex & & & 0.002 & & & 0.009 \\
\hline Male & 5.5 & $\begin{array}{l}4.6- \\
6.4\end{array}$ & & 9.1 & $\begin{array}{l}8.0- \\
10.2\end{array}$ & \\
\hline Female & 9.3 & $\begin{array}{l}8.1- \\
10.5\end{array}$ & & 16.8 & $\begin{array}{l}10.5- \\
23.1\end{array}$ & \\
\hline Smoking status & & & 0.084 & & & 0.024 \\
\hline Ever & 6.0 & $\begin{array}{l}5.3- \\
6.7\end{array}$ & & 9.1 & $\begin{array}{l}8.0- \\
10.2\end{array}$ & \\
\hline Never & 8.5 & $\begin{array}{l}4.0- \\
13.0\end{array}$ & & 16.8 & $\begin{array}{l}5.6- \\
28.0\end{array}$ & \\
\hline ECOG PS & & & $<0.001$ & & & $<0.001$ \\
\hline $0-1$ & 8.5 & $\begin{array}{l}7.9- \\
9.1\end{array}$ & & 15.0 & $\begin{array}{l}9.7- \\
20.3\end{array}$ & \\
\hline 2 & 3.1 & $\begin{array}{l}1.3- \\
4.9\end{array}$ & & 4.0 & $2.9-5.1$ & \\
\hline $\mathrm{aCCl}$ & & & 0.044 & & & 0.013 \\
\hline$<8$ & 8.0 & $\begin{array}{l}7.1- \\
8.9\end{array}$ & & 15.9 & $\begin{array}{l}9.1- \\
22.7\end{array}$ & \\
\hline$\geq 8$ & 5.5 & $\begin{array}{l}3.2- \\
7.8\end{array}$ & & 8.5 & $\begin{array}{l}6.5^{-} \\
10.5\end{array}$ & \\
\hline TNM stage & & & 0.217 & & & 0.663 \\
\hline III & 8.3 & $\begin{array}{l}7.8- \\
8.8\end{array}$ & & 8.5 & $\begin{array}{l}5.1- \\
11.9\end{array}$ & \\
\hline IV & 5.4 & $\begin{array}{l}4.1- \\
6.7\end{array}$ & & 10.5 & $\begin{array}{l}8.7- \\
12.3\end{array}$ & \\
\hline T stage & & & 0.007 & & & 0.631 \\
\hline T1-2 & 8.5 & $\begin{array}{l}7.4- \\
9.6\end{array}$ & & 9.2 & $\begin{array}{l}6.6- \\
11.8\end{array}$ & \\
\hline
\end{tabular}




\begin{tabular}{|c|c|c|c|c|c|c|}
\hline Factors & $\begin{array}{l}\text { mPFS } \\
\text { (months) }\end{array}$ & $\begin{array}{l}95 \% \\
\mathrm{Cl}\end{array}$ & $\begin{array}{l}P \\
\text { value }\end{array}$ & $\begin{array}{l}\text { moS } \\
\text { (months) }\end{array}$ & $95 \% \mathrm{Cl}$ & $\begin{array}{l}P \\
\text { value }\end{array}$ \\
\hline T3-4 & 5.4 & $\begin{array}{l}4.3- \\
6.5\end{array}$ & & 11.8 & $\begin{array}{l}9.1- \\
14.5\end{array}$ & \\
\hline N stage & & & 0.129 & & & 0.043 \\
\hline No-2 & 9.3 & $\begin{array}{l}4.4- \\
14.2\end{array}$ & & 17.5 & $\begin{array}{l}8.6- \\
26.4\end{array}$ & \\
\hline N3 & 6.0 & $\begin{array}{l}5.3- \\
6.7\end{array}$ & & 9.2 & $\begin{array}{l}7.5- \\
10.9\end{array}$ & \\
\hline Number of metastatic sites & & & 0.114 & & & 0.226 \\
\hline$<2$ & 8.3 & $\begin{array}{l}7.6- \\
9.0\end{array}$ & & 11.8 & $\begin{array}{l}6.2- \\
17.4\end{array}$ & \\
\hline$\geq 2$ & 5.4 & $\begin{array}{l}4.9- \\
5.9\end{array}$ & & 9.2 & $\begin{array}{l}7.7- \\
10.7\end{array}$ & \\
\hline Brain metastases & & & 0.851 & & & 0.506 \\
\hline Yes & 6.7 & $\begin{array}{l}3.3- \\
10.1\end{array}$ & & 8.6 & $\begin{array}{l}1.0- \\
16.2\end{array}$ & \\
\hline No & 6.0 & $\begin{array}{l}3.0- \\
9.0\end{array}$ & & 10.5 & $\begin{array}{l}8.8- \\
12.2\end{array}$ & \\
\hline Hepatic metastases & & & 0.010 & & & $<0.001$ \\
\hline Yes & 4.7 & $\begin{array}{l}3.2- \\
6.2\end{array}$ & & 5.4 & $1.2-9.6$ & \\
\hline No & 8.0 & $\begin{array}{l}6.1- \\
9.9\end{array}$ & & 15.0 & $\begin{array}{l}9.8- \\
20.2\end{array}$ & \\
\hline Osseous metastases & & & 0.238 & & & 0.287 \\
\hline Yes & 5.4 & $\begin{array}{l}4.9- \\
5.9\end{array}$ & & 9.1 & $\begin{array}{l}4.4- \\
13.8\end{array}$ & \\
\hline No & 7.7 & $\begin{array}{l}5.1- \\
10.3\end{array}$ & & 10.5 & $\begin{array}{l}6.6- \\
14.4\end{array}$ & \\
\hline $\begin{array}{l}\text { Pleural metastases / pleural } \\
\text { effusion }\end{array}$ & & & 0.132 & & & 0.700 \\
\hline Yes & 5.4 & $\begin{array}{l}4.0- \\
6.8\end{array}$ & & 9.2 & - & \\
\hline No & 8.0 & $\begin{array}{l}5.5- \\
10.5\end{array}$ & & 10.5 & $\begin{array}{l}7.9- \\
13.1\end{array}$ & \\
\hline Lung metastases & & & 0.849 & & & 0.912 \\
\hline
\end{tabular}




\begin{tabular}{|c|c|c|c|c|c|c|}
\hline Factors & $\begin{array}{l}\text { mPFS } \\
\text { (months) }\end{array}$ & $\begin{array}{l}95 \% \\
\mathrm{Cl}\end{array}$ & $\begin{array}{l}P \\
\text { value }\end{array}$ & $\begin{array}{l}\text { mOS } \\
\text { (months) }\end{array}$ & $95 \% \mathrm{Cl}$ & $\begin{array}{l}P \\
\text { value }\end{array}$ \\
\hline Yes & 5.1 & $\begin{array}{l}3.0- \\
7.2\end{array}$ & & 10.5 & $\begin{array}{l}3.6- \\
17.4\end{array}$ & \\
\hline No & 6.7 & $\begin{array}{l}4.1- \\
9.3\end{array}$ & & 9.2 & $\begin{array}{l}7.0- \\
11.4\end{array}$ & \\
\hline Baseline NSE & & & 0.006 & & & 0.051 \\
\hline$\leq 20 \mathrm{ng} / \mathrm{ml}$ & 8.5 & $\begin{array}{l}7.8- \\
9.2\end{array}$ & & 21.4 & - & \\
\hline$>20 \mathrm{ng} / \mathrm{ml}$ & 5.4 & $\begin{array}{l}4.6- \\
6.2\end{array}$ & & 9.2 & $\begin{array}{l}7.1- \\
11.3\end{array}$ & \\
\hline $\begin{array}{l}\text { Anlotinib initial } \\
\text { dose }\end{array}$ & & & 0.970 & & & 0.534 \\
\hline $10 \mathrm{mg}$ & 2.6 & $\begin{array}{l}0.0- \\
6.9\end{array}$ & & 8.6 & $\begin{array}{l}1.9- \\
15.3\end{array}$ & \\
\hline $12 \mathrm{mg}$ & 6.7 & $\begin{array}{l}4.3- \\
9.1\end{array}$ & & 10.5 & $\begin{array}{l}7.5- \\
13.5\end{array}$ & \\
\hline Plus radiotherapy & & & 0.002 & & & $<0.001$ \\
\hline Yes & 8.3 & $\begin{array}{l}6.6- \\
10.0\end{array}$ & & 16.8 & $\begin{array}{l}14.7- \\
18.9\end{array}$ & \\
\hline No & 4.2 & $\begin{array}{l}1.9- \\
6.5\end{array}$ & & 7.7 & $\begin{array}{l}3.9- \\
11.5\end{array}$ & \\
\hline Post-medication hypertension & & & 0.008 & & & $<0.001$ \\
\hline Yes & 8.5 & $\begin{array}{l}6.1- \\
10.9\end{array}$ & & 15.9 & $\begin{array}{l}14.0- \\
17.8\end{array}$ & \\
\hline No & 5.4 & $\begin{array}{l}3.4- \\
7.4\end{array}$ & & 8.3 & $\begin{array}{l}3.3- \\
13.3\end{array}$ & \\
\hline $\begin{array}{l}\text { Post-medication hand-foot } \\
\text { syndrome }\end{array}$ & & & 0.040 & & & 0.115 \\
\hline Yes & 8.5 & $\begin{array}{l}7.4- \\
9.6\end{array}$ & & 15.9 & $\begin{array}{l}9.9- \\
21.9\end{array}$ & \\
\hline No & 5.5 & $\begin{array}{l}4.2- \\
6.8\end{array}$ & & 9.2 & $\begin{array}{l}7.1- \\
11.3\end{array}$ & \\
\hline \multicolumn{7}{|c|}{$\begin{array}{l}\text { Abbreviations: PFS: progression-free survival; OS: overall survival; Cl: confidence interval; ECOG PS: } \\
\text { Eastern Cooperative Oncology Group Performance Status; aCCI: age-adjusted Charlson comorbidity } \\
\text { index; NSE: neuron specific enolase. }\end{array}$} \\
\hline Bold value represents $P$-value & & & & & & \\
\hline
\end{tabular}


Table 3

Multivariate Cox regression analysis of factors associated with PFS.

\begin{tabular}{|c|c|c|c|}
\hline Factors & HR & $95 \% \mathrm{Cl}$ & P-value \\
\hline Sex (Male vs. Female) & 6.05 & $1.74-20.98$ & 0.005 \\
\hline ECOG PS (2 vs. 0-1) & 8.34 & 2.54-27.39 & $<0.001$ \\
\hline $\mathrm{aCCl}(\geq 8 v s . \leq 8)$ & 1.51 & $0.74-3.06$ & 0.257 \\
\hline T stage (T3-4 vs. T1-2) & 3.82 & $1.59-9.18$ & 0.003 \\
\hline Hepatic metastases (Yes vs. No) & 0.67 & $0.30-1.49$ & 0.323 \\
\hline Baseline NSE (>20ng/ml vs. $\leq 20 \mathrm{ng} / \mathrm{ml})$ & 1.39 & $0.47-4.06$ & 0.551 \\
\hline Plus radiotherapy (Yes vs. No) & 0.98 & $0.30-3.23$ & 0.979 \\
\hline Post-medication hypertension (Yes vs. No) & 0.72 & $0.31-1.68$ & 0.450 \\
\hline Post-medication hand-foot syndrome (Yes vs. No) & 0.23 & $0.07-0.72$ & 0.012 \\
\hline \multicolumn{4}{|c|}{$\begin{array}{l}\text { Abbreviations: PFS: progression-free survival; HR: Hazard ratio; Cl: Confidence inter; ECOG PS: Eastern } \\
\text { Cooperative Oncology Group Performance Status; aCCl: age-adjusted Charlson comorbidity index; } \\
\text { NSE: neuron specific enolase. }\end{array}$} \\
\hline \multicolumn{4}{|l|}{ Bold value represents $P$-value $<0.05$. } \\
\hline
\end{tabular}

Table 4

Multivariate Cox regression analysis of factors associated with OS.

\begin{tabular}{|llll|}
\hline Factors & HR & $95 \%$ Cl & P-value \\
\hline Age ( $\geq 65$ vs. $<65)$ & 4.87 & $1.71-13.82$ & 0.003 \\
\hline Sex (Male vs. Female) & 0.88 & $0.13-5.83$ & 0.891 \\
\hline Smoking status (Ever vs. Never) & 2.52 & $0.51-12.49$ & 0.258 \\
\hline ECOG PS (2 vs. 0-1) & 11.26 & $2.49-50.84$ & 0.002 \\
\hline aCCl ( $\geq 8$ vs. $\leq 8)$ & 1.89 & $0.69-5.13$ & 0.213 \\
\hline N stage (N3 vs. N0-2) & 0.90 & $0.18-4.57$ & 0.899 \\
\hline Hepatic metastases (Yes vs. No) & 3.83 & $1.41-10.41$ & 0.008 \\
\hline Plus radiotherapy (Yes vs. No) & 0.73 & $0.17-3.04$ & 0.662 \\
\hline Post-medication hypertension (Yes vs. No) & 0.18 & $0.05-0.58$ & 0.005 \\
\hline $\begin{array}{l}\text { Abbreviations: OS: overall survival; HR: Hazard ratio; Cl: Confidence inter; ECOG PS: Eastern } \\
\text { Cooperative Oncology Group Performance Status; aCCl: age-adjusted Charlson comorbidity index. } \\
\text { Bold value represents } P \text {-value <0.05. }\end{array}$ & & \\
\hline
\end{tabular}




\section{Safety}

All of the 58 patients were available for safety profile. The incidence of treatment-related adverse reactions was $70.7 \%(41 / 58)$, and the incidence of grade 3 and above adverse reactions was $24.1 \%$ $(14 / 58)$ among the participants. Dose reductions due to adverse reactions were required for $16(27.6 \%)$ patients, and $7(12.1 \%)$ patients discontinued the treatment. There were no treatment-related deaths in this research. As shown in Table 5, the most common adverse reaction was myelosuppression (44.8\%), followed by hypertension (41.4\%), fatigue (34.5\%), gastrointestinal reaction (32.7\%), hand-foot syndrome $(24.1 \%)$, and so on. Notably, most of the adverse reactions were grade 1-2.

Table 5

Summary of adverse reactions.

\begin{tabular}{|llll|}
\hline Toxicity & All grades (\%) & Grade 1-2 (\%) & Grade $\geq 3$ (\%) \\
\hline Myelosuppression & $26(44.8)$ & $17(29.3)$ & $9(15.5)$ \\
\hline Hypertension & $24(41.4)$ & $21(36.2)$ & $3(5.2)$ \\
\hline Fatigue & $20(34.5)$ & $20(34.5)$ & $0(0.0)$ \\
\hline Gastrointestinal reaction & $19(32.7)$ & $17(29.3)$ & $2(3.4)$ \\
\hline Hand-foot syndrome & $14(24.1)$ & $13(22.4)$ & $1(1.7)$ \\
\hline Hyperlipemia & $11(19.0)$ & $11(19.0)$ & $0(0.0)$ \\
\hline Hemorrhage & $9(15.5)$ & $6(10.3)$ & $3(5.2)$ \\
\hline Transaminase elevation & $6(10.3)$ & $5(8.6)$ & $1(1.7)$ \\
\hline Hyponatremia & $6(10.3)$ & $6(10.3)$ & $0(0.0)$ \\
\hline Hyperbilirubinemia & $6(10.3)$ & $4(6.9)$ & $2(3.4)$ \\
\hline Hypophosphatemia & $5(8.6)$ & $4(6.9)$ & $1(1.7)$ \\
\hline Mucositis oral & $5(8.6)$ & $4(6.9)$ & $1(1.7)$ \\
\hline Rash & $4(6.9)$ & $4(6.9)$ & $0(0.0)$ \\
\hline Thyroid dysfunction & $3(5.2)$ & $3(5.2)$ & $0(0.0)$ \\
\hline Hypokalemia & $3(5.2)$ & $3(5.2)$ & $0(0.0)$ \\
\hline Proteinuria & $3(5.2)$ & $3(5.2)$ & $0(0.0)$ \\
\hline Hoarseness & $1(1.7)$ & $1(1.7)$ & $0(0.0)$ \\
\hline Arthralgia & $1(1.7)$ & $1(1.7)$ & $0(0.0)$ \\
\hline
\end{tabular}

\section{Discussion}


SCLC has an abnormally high proliferation rate, a strong tendency for early metastasis, and a bleak prognosis ${ }^{[2]}$. As the first-line standard treatment for ES-SCLC in the past 40 years, the PFS of platinumetoposide chemotherapy is about 5 months, and the median $O S$ is about 10 months ${ }^{[6]}$. Based on the IMpower133 and CASPIAN clinical trials, PD-L1 plus platinum-etoposide chemotherapy has become the new first-line standard therapy in recent years. Although, it only brought the OS benefit for 2 to 3 months ${ }^{[7}$, ${ }^{8]}$. Angiogenesis serves a pivotal role in tumor occurrence, invasion, and metastasis ${ }^{[16]}$. However, the efficacy of antiangiogenic therapy in SCLC is limited, such as bevacizumab, sorafenib, sunitinib and so on, except for anlotinib ${ }^{[17-20]}$. In China, anlotinib has been approved by CFDA as the third-line and above treatment for SCLC based on the ALTER 1202 study ${ }^{[12]}$. Several small sample size single arm phase II clinical trials of anlotinib combined with platinum-etoposide chemotherapy as the first-line treatment for ES-SCLC are being carried out in China and the preliminary results have shown the favorable clinical efficacy ${ }^{[13-15]}$.

Antiangiogenic therapy can improve drug delivery efficiency by opening the vascular normalization window, thus exerting a synergistic effect when combined with other regimens ${ }^{[21]}$. In addition, the nonoverlapping toxicity spectrum and excellent tolerance of anlotinib allow it to be used in combination with other drugs. In Kong T's clinical study, 20 ES-SCLC patients received anlotinib plus platinum-etoposide chemotherapy as the first-line therapy, the median PFS was 10.0 months, and the median OS was 15.0 months ${ }^{[13]}$. Similarly, in Deng P's study, the median PFS and OS were 9.4 and 13.9 months, respectively ${ }^{[15]}$. Supported by these encouraging preliminary results, phase III clinical trials have already begun in China. In this real-world study, the median PFS was 6.0 months, the median OS was 10.5 months, the ORR was $58.6 \%$, and the DCR was $89.6 \%$. Our results are similar to the efficacy of traditional platinum-etoposide chemotherapy. But 20 (34.5\%) patients with ECOG PS 2 were included in this study. In contrast, there were no patients with ECOG PS $>1$ in these clinical trials. The median PFS and OS of patients with ECOG PS 0-1 in this study were 8.5 and 15.0 months, respectively. Multivariate Cox regression analysis showed that ECOG PS was the independent influencing factor of PFS and OS. This result showed better efficacy compared with traditional chemotherapy and PD-L1 plus chemotherapy, and the OS was similar to the clinical studies of anlotinib plus platinum-etoposide chemotherapy. Since there is no control group in our study, the efficacy of combination therapy still requires further verification by prospective studies with larger sample size.

ES-SCLC patients first receive chemotherapy to control the spread of metastasis. Subsequently, chest radiotherapy is recommended to control local lesions for patients who achieve CR or PR after chemotherapy ${ }^{[22]}$. Some studies found that antiangiogenic therapy can increase the local oxygen partial pressure and oxygen content of tumor tissue, inhibit the angiogenesis induced by radiotherapy, and play the role of radiotherapy sensitization ${ }^{[23]}$. In our study, patients combined with radiotherapy had more extended PFS ( 8.3 vs. 4.2 months, $P=0.002)$ and OS (16.8 vs. 7.7 months, $P<0.001)$ benefits in univariate analysis. However, there were no statistical differences in multivariate analysis. 
Hypertension and hand-foot syndrome are the most common adverse reactions of anlotinib. Interestingly, more extended PFS benefits were observed in ES-SCLC patients with post-medication hypertension or hand-foot syndrome in Song PF's study ${ }^{[24]}$. In this research, patients with post-medication hypertension (8.5 vs. 5.4 months, $P=0.008$ ) and hand-foot syndrome ( 8.5 vs. 5.5 months, $P=0.040$ ) had longer PFS benefits in univariate analysis. Additionally, we also found that patients with post-medication hypertension ( 15.9 vs. 8.3 months, $P<0.001$ ) had longer OS benefits. Multivariate analysis showed that post-medication hand-foot syndrome (yes $v s$. no: $\mathrm{HR}=0.23,95 \% \mathrm{Cl} 0.07-0.72, P=0.012$ ) was the independent predictor of PFS, and post-medication hypertension (yes vs. no: $\mathrm{HR}=0.18,95 \% \mathrm{Cl} 0.05-0.58, P$ $=0.005)$ was the independent predictor of OS.

Furthermore, we observed that sex (male vs. female: $\mathrm{HR}=6.05,95 \% \mathrm{Cl} 1.74-20.98, P=0.005$ ) and T stage (T3-4 vs. T1-2: $\mathrm{HR}=3.82,95 \% \mathrm{Cl} 1.59-9.18, P=0.003$ ) were the independent influencing factors of PFS. Age ( $\geq 65$ vs. $<65$ : $\mathrm{HR}=4.87,95 \% \mathrm{Cl} 1.71-13.82, P=0.003$ ) and hepatic metastases (yes vs. no: $\mathrm{HR}=3.83,95 \% \mathrm{Cl}$ 1.41-10.41, $P=0.008$ ) were associated with OS in multivariate Cox regression analysis. However, only 11 (19.0\%) female patients were included in our study, which might influence the result.

In this research, the toxicity of anlotinib plus platinum-etoposide chemotherapy was generally well tolerated. The grade 3 and above adverse reactions were manageable with dose reduction or drug discontinuation. Similar to previous research, myelosuppression was the most frequent adverse reaction $^{[13-15]}$. As the most common adverse reactions of anlotinib, the incidence of hypertension and hand-foot syndrome were $41.1 \%$ and $24.1 \%$, respectively. There were no new anlotinib-related adverse reactions observed in this study, and the toxic profile was similar to other studies of anlotinib in SCLC ${ }^{[12]}$. The incidence of adverse reactions in this research might be lower than actual data in the real world because of the bias of the retrospective study.

This study provides real-world data of anlotinib combined with platinum-etoposide chemotherapy as the first-line treatment for ES-SCLC at the first time. However, as a retrospective study, our research still has some unavoidable limitations. First, as a real-world study, the Chinese undiversified population and small sample size might affect the universality of the results. Thus, future study with larger sample size is needed. Second, the proportion of female patients is low, which may affect the representation of the population. Last but not least, since the dosage was determined by different doctors according to the actual situation of patients, and this may affect the efficacy.

\section{Conclusion}

Anlotinib combined with platinum-etoposide chemotherapy as the first-line treatment for ES-SCLC appears to be effective and well-tolerated in the real-world, especially in patients with ECOG PS 0-1. Patients with post-medication hypertension and hand-foot syndrome may confer superior prognosis.

\section{Declarations}


Acknowledgements

We would like to thank Xijing Hospital of Air Force Military Medical University, Xianyang Central Hospital, Shaanxi Nuclear Industry 215 Hospital, Hanzhong Central Hospital and Baoji Traditional Chinese Medicine Hospital for providing the clinical data.

\section{Authors' contributions}

YY contributed to the concept and design of the research; HRZ established the database; HRZ, AMJ, and $H G$ conducted statistical analysis; $H R Z$ and AMJ wrote the first draft of the manuscript; $H G, N L, X Q Z, X F$, $Z P R, T T, X L$ and $Y Y$ reviewed and edited the manuscript. All authors participated in the revision of the manuscript, read and approved the submitted version.

\section{Funding}

This study was funded by International Cooperation Project in Science and Technology of Shaanxi Province (No. 2019KW-074), Nation Natural Science Funding of China (No. 82002437), and Shaanxi Province Technology Innovation Team (No. 2021TD-44).

\section{Availability of data and materials}

The original contributions presented in the study are included in the article / supplementary materials. Further inquiries can be directed to the corresponding authors.

\section{Ethical approval}

This study was approved by the Ethics Committee of the First Affiliated Hospital of Xi'an Jiaotong University (No. XJTU1AF2019LSK-132) and conformed to the provisions of the Declaration of Helsinki. Since the study is a retrospective study, it did not damage the health and interests of patients, and protected their privacy and personal information, the study is exempted from informed consent.

\section{Consent for publication}

Informed consent was obtained from the authors.

\section{Competing interests}

The authors declare that they have no competing interests.

\section{References}

1. Herbst RS, Heymach JV, Lippman SM (2008) Lung cancer[J]. N Engl J Med 359(13):1367-1380

2. Rudin CM, Brambilla E, Faivre-Finn C et al (2021) Small-cell lung cancer[J]. Nat Rev Dis Primers $7(1): 3$ 
3. Yang S, Zhang Z, Wang Q (2019) Emerging therapies for small cell lung cancer[J]. J Hematol Oncol 12(1):47

4. Carney DN (2002) Lung cancer-time to move on from chemotherapy[J]. N Engl J Med 346(2):126128

5. Van Meerbeeck JP, Fennell DA, De Ruysscher DK (2011) Small-cell lung cancer[J]. Lancet 378(9804):1741-1755

6. Farago AF, Keane FK (2018) Current standards for clinical management of small cell lung cancer[J]. Transl Lung Cancer Res 7(1):69-79

7. Liu SV, Reck M, Mansfield AS et al (2021) Updated Overall Survival and PD-L1 Subgroup Analysis of Patients With Extensive-Stage Small-Cell Lung Cancer Treated With Atezolizumab, Carboplatin, and Etoposide (IMpower133)[J]. J Clin Oncol 39(6):619-630

8. Paz-Ares L, Dvorkin M, Chen Y et al (2019) Durvalumab plus platinum-etoposide versus platinumetoposide in first-line treatment of extensive-stage small-cell lung cancer (CASPIAN): a randomised, controlled, open-label, phase 3 trial[J]. Lancet 394(10212):1929-1939

9. Ferrara N, Gerber HP, Lecouter J (2003) The biology of VEGF and its receptors[J]. Nat Med 9(6):669676

10. Zhan P, Wang J, Lv XJ et al (2009) Prognostic value of vascular endothelial growth factor expression in patients with lung cancer: a systematic review with meta-analysis[J]. J Thorac Oncol 4(9):10941103

11. Shen G, Zheng F, Ren D et al (2018) Anlotinib: a novel multi-targeting tyrosine kinase inhibitor in clinical development[J]. J Hematol Oncol 11(1):120

12. Cheng Y, Wang Q, Li K et al (2021) Anlotinib vs placebo as third- or further-line treatment for patients with small cell lung cancer: a randomised, double-blind, placebo-controlled Phase 2 study[J]. $\mathrm{Br} \mathrm{J}$ Cancer 125(3):366-371

13. Kong T, Chen L, Duan F et al (2021) Efficacy and safety analysis of anlotinib combined with etoposide plus cisplatin/carboplatin as first-line therapy for extensive-stage small cell lung cancer (SCLC): The final results from a phase II single-arm trial[J].Journal of Clinical Oncology, 39(15)

14. Han B, Zhang W, Zhang B et al (2021) Anlotinib Plus Etoposide and Carboplatin as First-Line Treatment for Extensive-Stage Small Cell Lung Cancer: A Single Arm Phase II Trial[J]. Journal of Thoracic Oncology 16(3):S503-S503

15. Deng P, Yang H, Chen $\mathrm{C}$ et al (2020) The efficacy and safety profile of anlotinib with etoposide plus cisplatin/carboplatin in treatment-naive extensive-stage small cell lung cancer(SCLC) patients: Results from a phase II single-arm trial[J].Journal of Clinical Oncology, 38(15)

16. Hanahan D, Weinberg RA (2011) Hallmarks of cancer: the next generation[J]. Cell 144(5):646-674

17. Pujol JL, Lavole A, Quoix E et al (2015) Randomized phase II-III study of bevacizumab in combination with chemotherapy in previously untreated extensive small-cell lung cancer: results from the IFCT-0802 trialt[J]. Ann Oncol 26(5):908-914 
18. Sharma N, Pennell N, Nickolich M et al (2014) Phase II trial of sorafenib in conjunction with chemotherapy and as maintenance therapy in extensive-stage small cell lung cancer[J]. Invest New Drugs 32(2):362-368

19. Han JY, Kim HY, Lim KY et al (2013) A phase II study of sunitinib in patients with relapsed or refractory small cell lung cancer[J]. Lung Cancer 79(2):137-142

20. Montanino A, Manzo A, Carillio G et al (2021) Angiogenesis Inhibitors in Small Cell Lung Cancer[J]. Front Oncol 11:655316

21. Alshangiti A, Chandhoke G, Ellis PM (2018) Antiangiogenic therapies in non-small-cell lung cancer[J]. Curr Oncol 25(Suppl 1):S45-s58

22. Picardi C, Caparroti F, Di Maio M et al (2019) Prophylactic cranial irradiation in extensive disease small cell lung cancer: An endless debate[J]. Crit Rev Oncol Hematol 143:95-101

23. Ansiaux R, Dewever J, Grégoire $V$ et al (2009) Decrease in tumor cell oxygen consumption after treatment with vandetanib (ZACTIMA; ZD6474) and its effect on response to radiotherapy[J]. Radiat Res 172(5):584-591

24. Song PF, Xu N, Li Q (2020) Efficacy and Safety of Anlotinib for Elderly Patients with Previously Treated Extensive-Stage SCLC and the Prognostic Significance of Common Adverse Reactions[J]. Cancer Manag Res 12:11133-11143

\section{Figures}
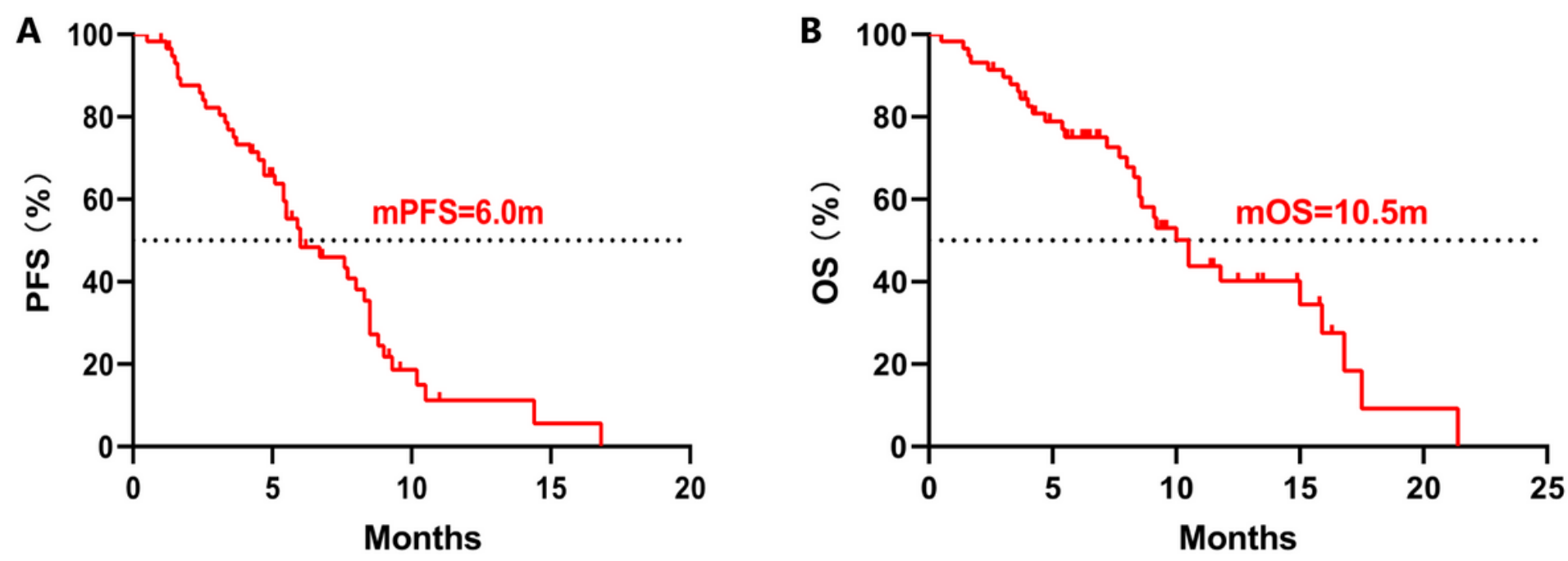

Figure 1

Kaplan-Meier curves of all patients. (A) The Kaplan-Meier curve of PFS; (B) The Kaplan-Meier curve of OS. PFS: progression-free survival; OS: overall survival. 


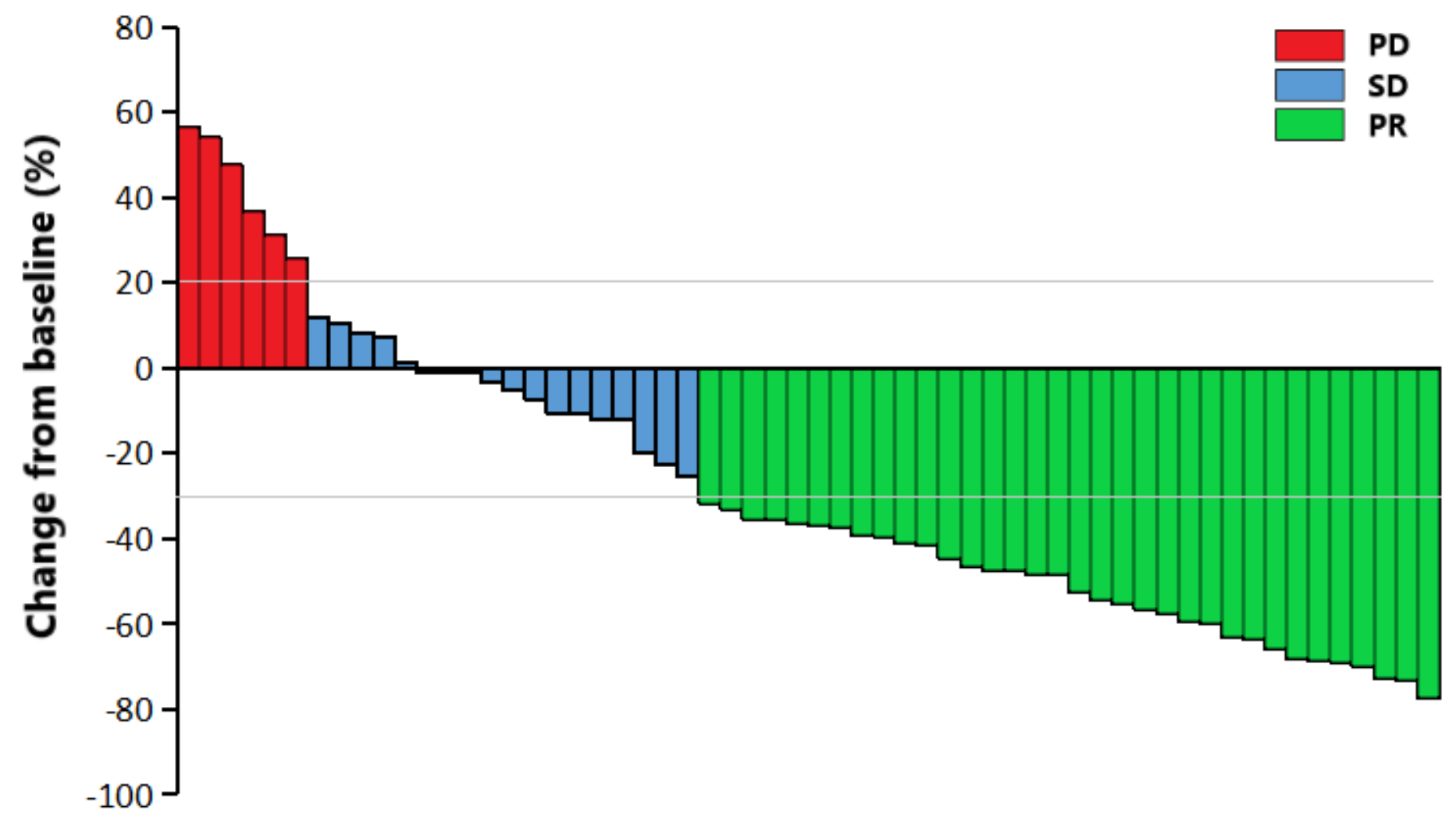

Figure 2

The waterfall plot of tumor best response compared with baseline measurable lesions. PR: partial response; SD: stable disease; PD: progressive disease. 

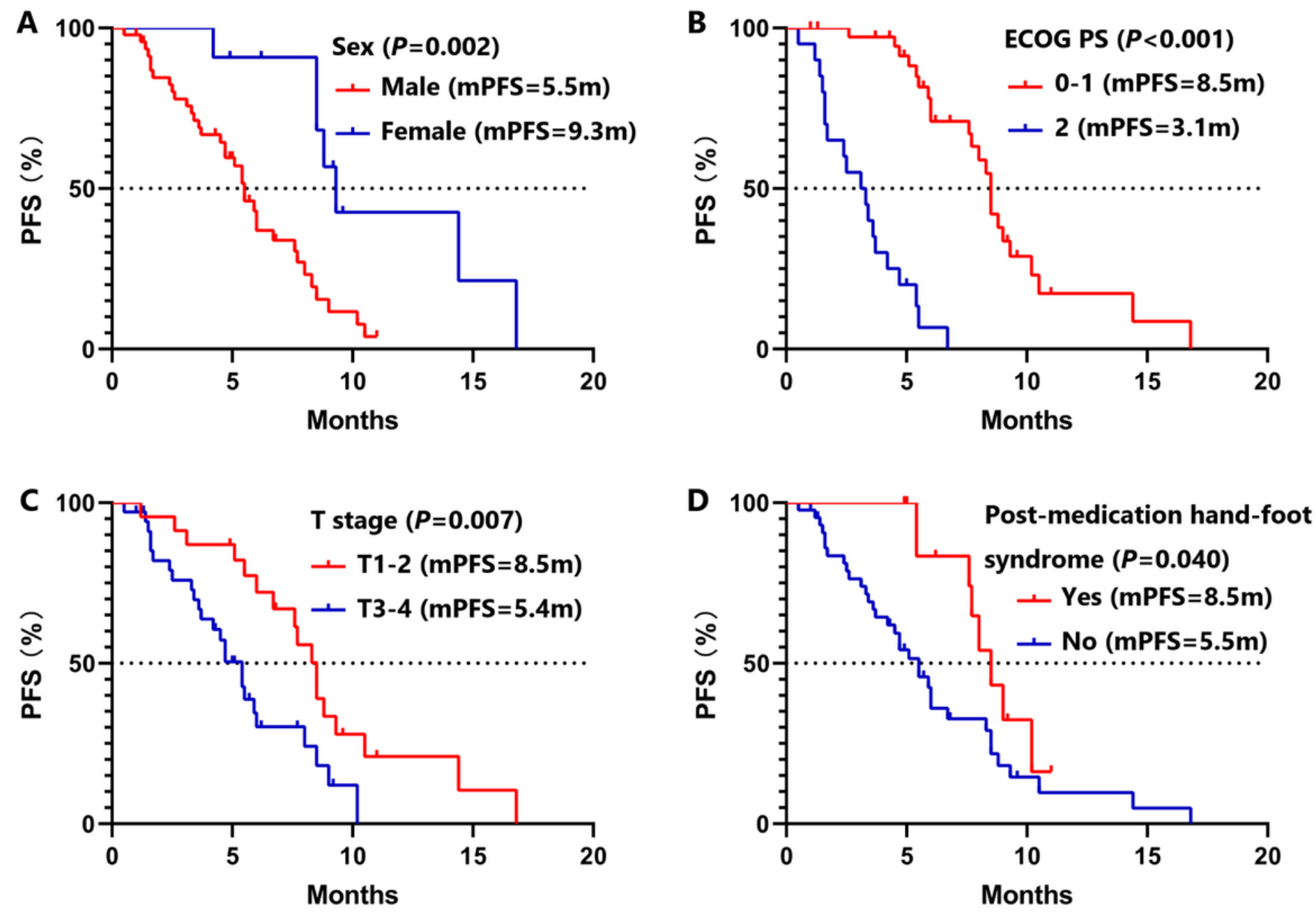

Figure 3

Kaplan-Meier curves of PFS in multivariate Cox regression analysis. (A) stratified by sex; (B) stratified by ECOG PS; (C) stratified by T stage; (D) stratified by post-medication hand-foot syndrome. PFS: progression-free survival; ECOG PS: Eastern Cooperative Oncology Group Performance Status. 

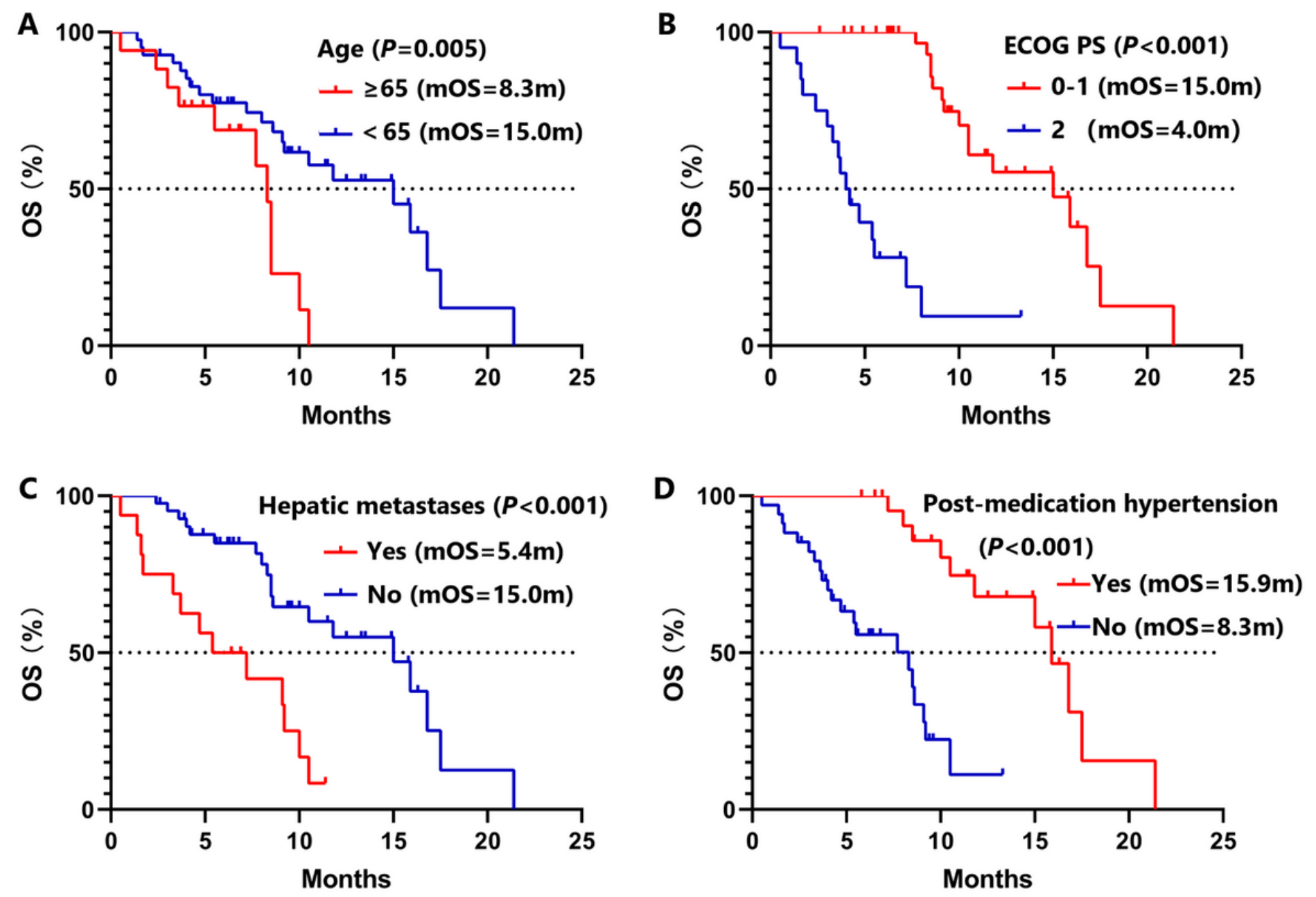

Figure 4

Kaplan-Meier curves of OS in multivariate Cox regression analysis. (A) stratified by age; (B) stratified by ECOG PS; (C) stratified by hepatic metastases; (D) stratified by post-medication hypertension. OS: overall survival; ECOG PS: Eastern Cooperative Oncology Group Performance Status. 